\title{
Vertical Flow in Viscous Thin Films
}

Khidr M.S. Khidr

College of Computer Science and Mathematics

University of Mosul, Iraq
Hikmat Sh. Mustafa

College of Education

Received on: 22/04/2007

Accepted on: 15/08/2007

\section{ABSTRACT}

The aim of this paper is to investigate the vertical flow in thin films of an incompressible liquids with no inertia force. Continuity equation and Navier-Stokes equations are used to obtain the equation that governs this type of flow, this equation is solved by using numerical methods to find the thickness of film.

Keywords: flow, thin films, incompressible liquids, Continuity equation, Navier-Stokes equations.

$$
\text { الجريان العمودي في الأغثية الرقيقة اللزجة }
$$

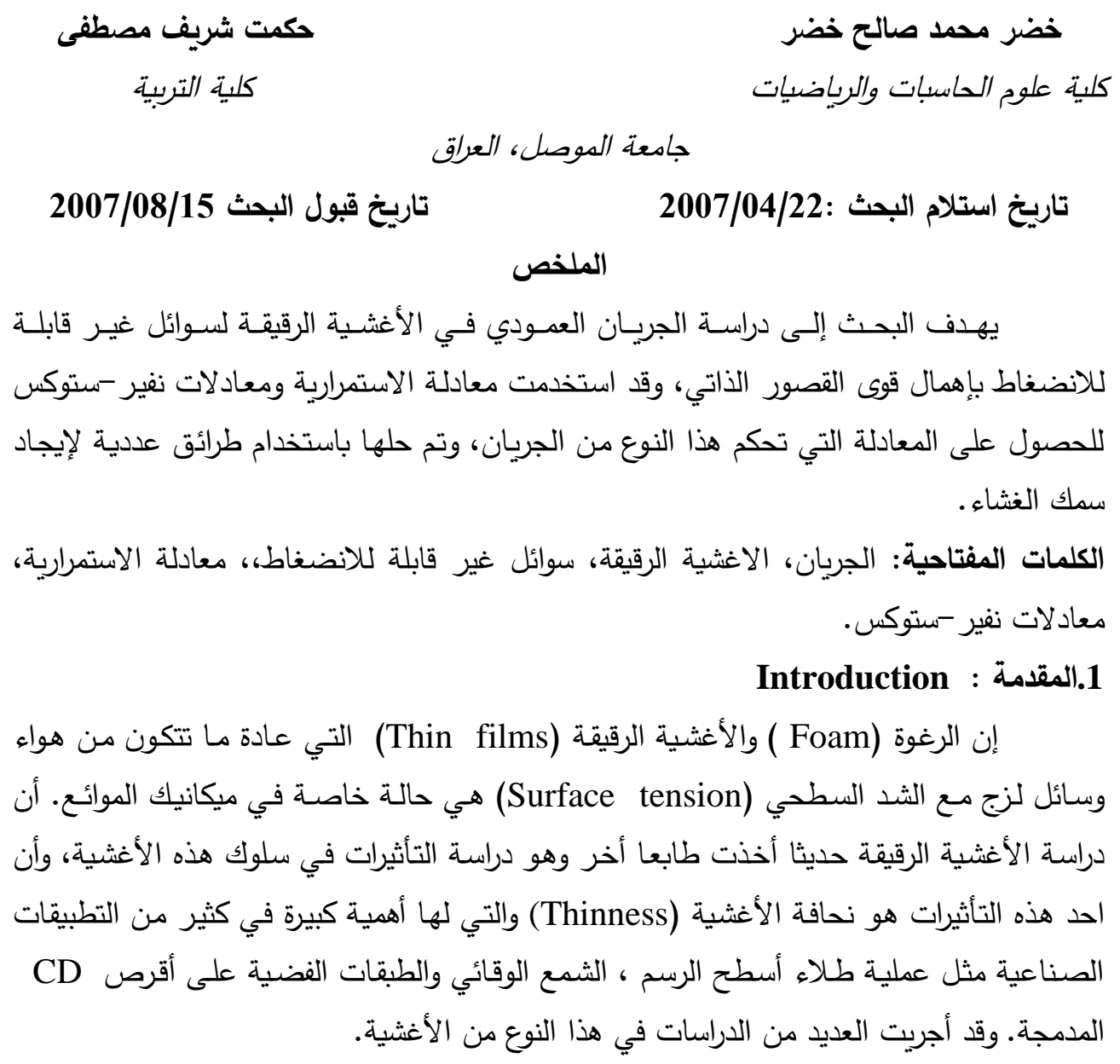


درس (Tuck, E.O. and L.W. Schwartz, 1990) الطرائق العددية لحل بعض

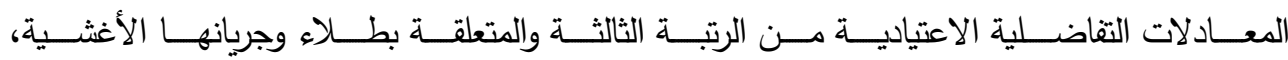
ودرس (Moriarty, J.A. and L.W.Shwartz,1991) الانتثار غير المستقر للأغشية الرقيقة السائلة مع شد سطحي صغير . كما درس (Majeed,M.A.S,2002) الجريـان اللازمني في الأغشية الرقيقة بإهـال قوى القصور الذاتي.

(Richard B.; Olivier, D.; Marcio, G.;Andrew T.; Haris W. وقام كل من ) بدراسة الجريان العمودي للأغشية الرقيقة في حالة سائلين، كما درس (and Kerianne Y.

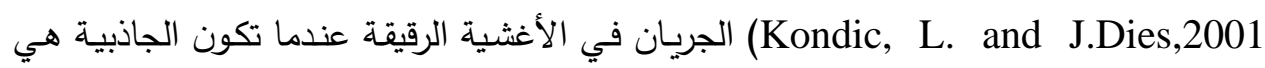
المسؤولة عن الجريان وتبين بأن خط الاتصال يكون غير مستقر ، ودرس (Braum,R.J.,2002) جريان الغشاء السائل الرقيق بإهمال قوى القصور الذاتي، كما درس (Boo;D,N.B.Morley and (الطرق العددية في جريان الأغشية الساقطة على شكل موجة باستخدام طريقة

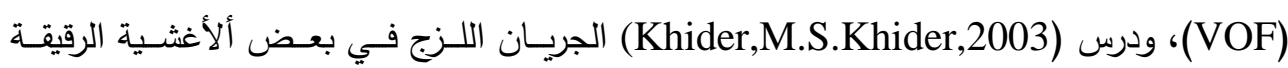
السائلة المائلة. وفي بحثنا هذا سوف نتطرق إلى دراسة الجريان العمودي في الأغشية الرقيقة اللزجة على سطح صلب.

Governing equations : المعادلات التي تحكم الجريان ليكن (u,v) الاتجاهين y,x على الترتيب و p يمثل الضغط (الضغط الداخلي للسائل).

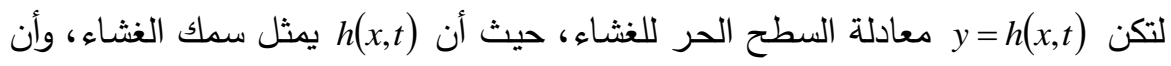
الجريان عمودي نحو الأسفل باتجاه الاحداثي x وكما مبين في الثكل (1,1 ) .

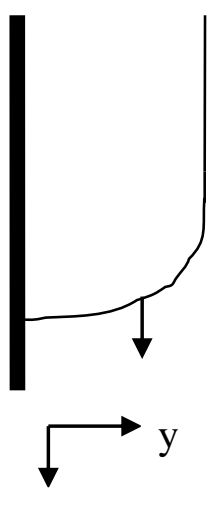


الثكل (1,1)

مقطع طولى لغشاء رقيق يجري على سطح صلب

نحصر اهتمامنا بالحالة التي يكون فيها 1>> $\left(\frac{\partial h}{\partial x}\right)$

الحد ${ }^{2}\left(\frac{\partial h}{\partial x}\right)^{2}$

$k=\frac{\frac{\partial^{2} h}{\partial x^{2}}}{\left[1+\left(\frac{\partial h}{\partial x}\right)^{2}\right]^{\frac{3}{2}}}$

فتصبح بالصيغة الآتية:-

$k=\frac{\partial^{2} h}{\partial x^{2}}$

إن المعادلات التي تحكم الجريان في الغشاء العمودي ولنظام ثنائي البعد هي معادلة الاستمرارية والتي لها الصيغة الآتية:-

$\frac{\partial u}{\partial x}+\frac{\partial v}{\partial y}=0$

ومعادلات حفظ الزخم معادلات (نافير ستوكس) في الاتجاهين y,x وعلى الترتيب:-

$\rho\left(\frac{\partial u}{\partial t}+u \frac{\partial u}{\partial x}+v \frac{\partial u}{\partial y}\right)=-\frac{\partial p}{\partial x}+\mu\left(\frac{\partial^{2} u}{\partial x^{2}}+\frac{\partial^{2} u}{\partial y^{2}}\right)+\rho g_{x}$

$\rho\left(\frac{\partial v}{\partial t}+u \frac{\partial v}{\partial x}+v \frac{\partial v}{\partial y}\right)=-\frac{\partial p}{\partial y}+\mu\left(\frac{\partial^{2} v}{\partial x^{2}}+\frac{\partial^{2} v}{\partial y^{2}}\right)+\rho g_{y}$

حيث أن م يمثل الكثافة ، $\mu$ اللزوجة ، و التعجيل الأرضي.

وباستخدام نظرية التزييت]1] فأن معادلتي الزخم (2.5) و و(2.6) تختزلان إلى الصيغة الآتية:-

$\frac{\partial p}{\partial x}=\mu \frac{\partial^{2} u}{\partial y^{2}}+\rho g$

$\frac{\partial p}{\partial y}=\mu\left(\frac{\partial^{2} v}{\partial x^{2}}+\frac{\partial^{2} v}{\partial y^{2}}\right)-\rho g$

Boundary conditions : الثروط الحدودية

1. شرط جهد القص : (Tangential stress conditions) على السطح الحر للغثاء عندما فأن 
$\tau=\mu\left(\frac{\partial u}{\partial y}\right)_{s}=0$

حيث أن : s s تمثل القيم على السطح الحر للغثاء .

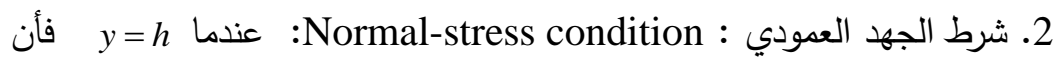

$p=-\sigma \frac{\partial^{2} h}{\partial x^{2}}$

حيث أن $\sigma$ يمثل الثد السطحي.

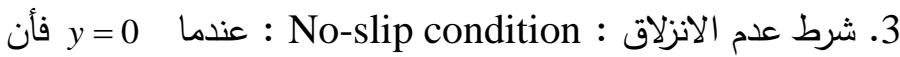

$u=0$

$$
\left.\begin{array}{ll}
x \rightarrow-\infty & \Rightarrow h \rightarrow 1 \\
x \rightarrow+\infty & \Rightarrow h \rightarrow+\infty
\end{array}\right\}
$$

باشتقاق الثرط الحدي (3.3) بالنسبة إلى xx نحصل على ما يأتي:-

$\frac{\partial p}{\partial x}=-\sigma \frac{\partial^{3} h}{\partial x^{3}}$

بمقارنة المعادلة (3.6) مع المعادلة (2.7) نحصل على ما يأتي :-

$\mu \frac{\partial^{2} u}{\partial y^{2}}+\rho g=-\sigma \frac{\partial^{3} h}{\partial x^{3}}$

بتكامل المعادلة (3.7) بالنسبة إلى y نحصل على ما يأتي:-

$\mu \frac{\partial u}{\partial y}=-\rho g y-\sigma \frac{\partial^{3} h}{\partial x^{3}} y+f(x, t)$

حيث أن

بتطبيق الثرط الحدي (3.2) في المعادلة (3.8) عندما h= y نحصل على ما يأتي:$f(x, t)=\rho g h+\sigma \frac{\partial^{3} h}{\partial x^{3}} h$

بتعويض المعادلة (3.9) في المعادلة (3.8) نحصل على ما يأتي :-

$\mu \frac{\partial u}{\partial y}=-\rho g y-\sigma \frac{\partial^{3} h}{\partial x^{3}} y+\rho g h+\sigma \frac{\partial^{3} h}{\partial x^{3}} h$

$\frac{\partial u}{\partial y}=\left(\frac{\rho g}{\mu}+\frac{\sigma}{\mu} \frac{\partial^{3} h}{\partial x^{3}}\right)(h-y)$

بتكامل المعادلة (3.11) بالنسبة إلى y نحصل على ما يأتي :- 


$$
\begin{aligned}
& u(x, y, t)=\left(\frac{\rho g}{\mu}+\frac{\sigma}{\mu} \frac{\partial^{3} h}{\partial x^{3}}\right)\left(h y-\frac{y^{2}}{2}\right)+g(x, t) \\
& \text { بتطبيق الشرط الحدي (3.4) في المعادلة (3.12) نحصل على ما يأتي :- }
\end{aligned}
$$

$$
g(x, t)=0
$$

وهكذا فأن المعادلة (3.12) تصبح بالصيغة الآتية:-

$$
u(x, y, t)=\left(\frac{\rho g}{\mu}+\frac{\sigma}{\mu} \frac{\partial^{3} h}{\partial x^{3}}\right)\left(h y-\frac{y^{2}}{2}\right)
$$

إن الصيغة التكاملية لمعادلة حفظ الكتلة تعطى بالصيغة [7]:-

$$
\frac{\partial h}{\partial t}=-\frac{\partial Q}{\partial x}=-\frac{\partial}{\partial x} \int_{0}^{h} u(x, y, t) d y
$$

حيث أن Q يمثل المعدل الحجمي للجريان. بتعويض المعادلة (3.14) في المعادلة(3.15) نحصل على ما ئاتي :-

$$
\frac{\partial h}{\partial t}=-\frac{\partial}{\partial x} \int_{0}^{h}\left(\frac{\rho g}{\mu}+\frac{\sigma}{\mu} \frac{\partial^{3} h}{\partial x^{3}}\right)\left(h y-\frac{y^{2}}{2}\right) d y
$$

$$
\frac{\partial h}{\partial t}=-\frac{\partial}{\partial x}\left(\frac{\rho g}{\mu}+\frac{\sigma}{\mu} \frac{\partial^{3} h}{\partial x^{3}}\right) \frac{h^{3}}{3}
$$

$$
3 \mu \frac{\partial h}{\partial t}=-\frac{\partial}{\partial x}\left(\rho g h^{3}+\sigma h^{3} \frac{\partial^{3} h}{\partial x^{3}}\right)
$$

والمعادلة ( 3.18) تمثل التوازن بين ثلاث قوى (اللزوجة،الثد السطحي والتعجيل الأرضي).

Non-dimensional : المتغيرات اللابعدية (4.1)

تعرف المتغيرات x,h بدلالة المتغيرات اللابعدية بالصيغة[7] :-

$$
\begin{aligned}
& h=\bar{h} H, \quad x=\bar{x} L, \quad t=\bar{t} T \\
& \text { وأن }
\end{aligned}
$$

بتعويض المتغيرات اللابعدية(4.2) في المعادلة( 3.18) نحصل على ما يأتي :-

$$
\frac{\partial \bar{h}}{\partial \bar{t}}=-\frac{\partial}{\partial \bar{x}}\left(\bar{h}^{3}+\varepsilon^{3} \bar{h}^{3} \frac{\partial^{3} \bar{h}}{\partial \bar{x}^{3}}\right)
$$




$$
\begin{aligned}
& \text { حيث أن } \\
& \text { وباسقاط الرمز (-) من المعادلة (4.3) فأنها تصبح بالصيغة الآتية:- } \\
& \frac{\partial h}{\partial t}=-\frac{\partial}{\partial x}\left(h^{3}+h^{3} \frac{\partial^{3} h}{\partial x^{3}}\right) \\
& \text { في حالة اللازمني أي عندما (4h }=0 \text { فأن المعادلة(4.4) تصبح بالصيغة :- } \\
& \frac{d}{d x}\left(h^{3}+h^{3} \frac{\partial^{3} h}{\partial x^{3}}\right)=0 \\
& h^{3} h^{\prime \prime \prime}+h^{3}=A \\
& \text { بتكامل المعادلة(4.5) بالنسبة إلى x نحصل على ما يأتي :- } \\
& h^{\prime \prime \prime}=-1+\frac{A}{h^{3}} \\
& \text { حيث أن A ثابت التكامل . }
\end{aligned}
$$

حيث أن a ثابت اختياري صغير جدا لموازنة العلاقة (4.8). لحل المعادلة(4.7) نحولها إلى نظام المعادلات التفاضلية وذلك بتخفيض رتبة المعادلة

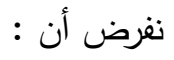

$h_{1}=h \rightarrow h_{1}=h^{\prime}=h_{2}$

$h_{2}=h^{\prime} \rightarrow h_{2}^{\prime}=h^{\prime \prime}=h_{3}$

$h_{3}=h^{\prime \prime} \rightarrow h_{3}^{\prime}=h^{\prime \prime \prime}=-1+\frac{A}{h^{3}}$

وبتطبيق الثـرط الابتدائي(4.8) عندما 0 = x وباستخدام طريقـة رنـج-كوتـا مـ الرتبـة

الرابعة في نظام الـ MATLAB نحصل على الحلول الآتية وكما موضحة في الثكل (1.2 )

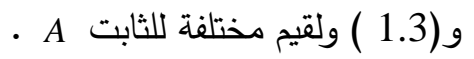




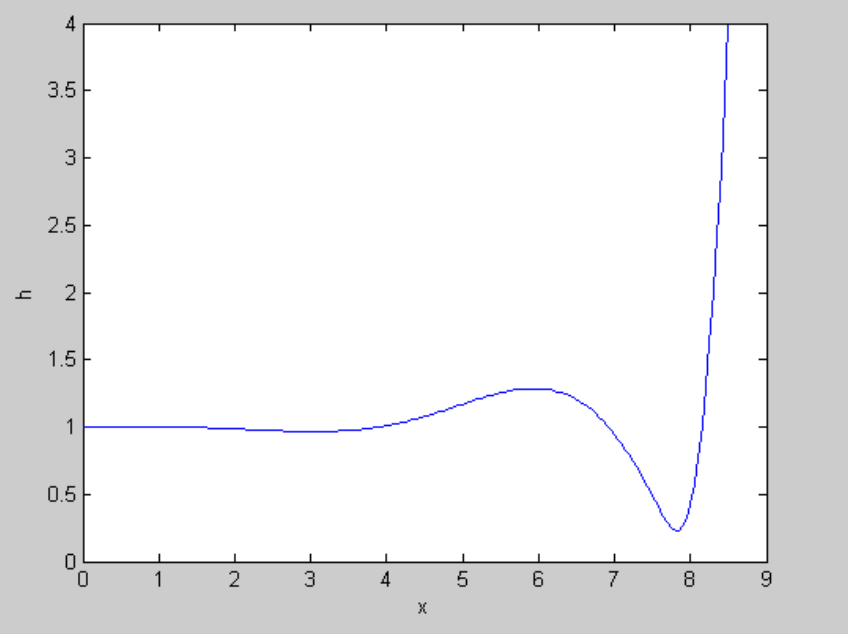

الثكل (1.2)

يمثل منحني الحل للمستوي (x,h) للمعادلة (4.7) عندما 1 =

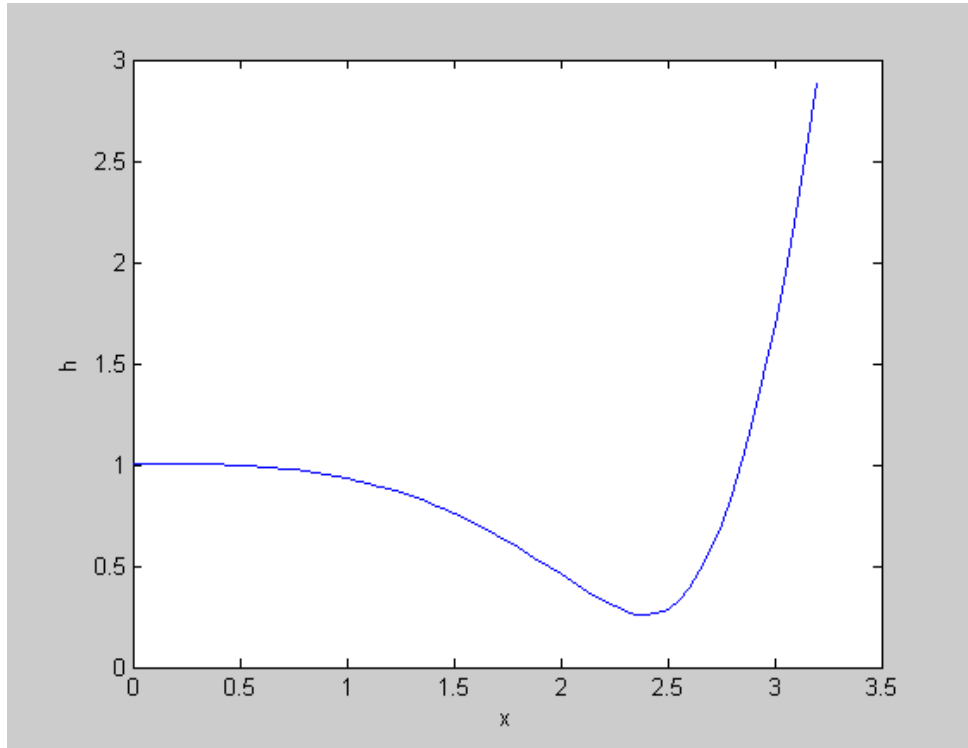

الثكل (1.3)

يمثل منحني الحل للمستوي (x,h) للمعادلة (4.7) عندما 0.5 
خضر محمد صالح و حكمت شربف مصطفى

\section{Conclusion : الاستنتاجات}

في هذا البحث تمت دراسـة جريان الاغشية الرقيقة اللزجة بصورة عمودية في نظام ثنائي

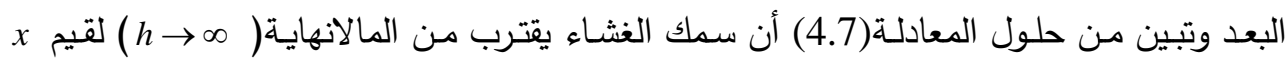

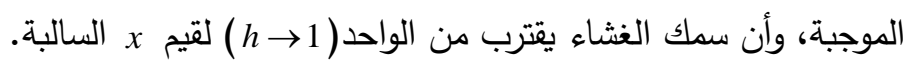




\section{المصادر}

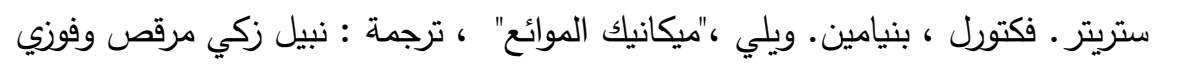

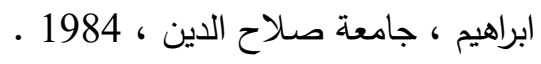

[2] Braum,R.J. (2002). "Thin fluid film drainage" $6^{\text {th }}$ PIMS Graduate Industrial Math Modelling Camp, Version of December 4.

[3] Gao;D,N.B.Morley and,V.Dhir, (2003) "Numerical simulation of wavy falling film flow using VOF method" Journal of computational Phyiscs 192 PP.624-642.

[4] Khider,M.S.Khider, (2003) "Viscous flow in certain inclined thin liquid films" M.Sc.thesis,University of Mosul.

[5] Kondic, L. and J.Dies, (2001) "Pattern formation in the flow of thin films down an incline: Constant flux configuration" Phys. Fluids, Vol 13, No 11.

[6] Majeed,M.A.S, (2002) "Steady flow in thin liquid films with negligible inertia" M.Sc.thesis, University of Mosul.

[7] Moriarty, J.A. and L.W.Shwartz. (1991)." Unsteady spreading of thin liquid films with small surface tension" Phys. Fluids A 3(5),May.

[8] Richard B.; Olivier, D.; Marcio, G.;Andrew T.; Haris W and Kerianne Y, "Vertical draining of thin films:Two fluids case".

[9] Schwartz, L.W. and R.V. Roy (1999) "Modeling draining flow in mobile and immobile soap films" Journal of Colloid and interface Science 218,pp309-323.

[10] Tuck, E.O. and L.W.Schwartz, (1990) "A Numerical and asymptotic study of some third-order ordinary differential equations relevant to draining and coating flows" SIM,Vol 32, No.3,pp.453-469. 